\title{
Interest in quantitative genetics of Dutt's and Deak's methods for numerical computation of multivariate normal probability integrals
}

\author{
V. DUCROCQ, J.J. COLLEAU
}

I.N.R.A., Station de Génétique quantitative et appliquée

Centre National de Recherches Zootechniques, F 78350 Jouy-en-Josas

\begin{abstract}
Summary
Numerical computation of multivariate normal probability integrals is often required in quantitative genetic studies. In particular, this is the case for the evaluation of the genetic superiorities after independent culling levels selection on several correlated traits, for certain methods used to analyse discrete traits and for some studies on selection involving a limited number of candidates.

Dutt's and Deak's methods can satisfy most of the geneticist's needs. They are presented in this paper and their precision is analysed in detail. It appears that Dutt's method is remarkably precise for dimensions 1 to 5 , except when truncation points or correlation coefficients between traits are very high in absolute value. Deak's method, less precise, is better suited for higher dimensions (6 to 20) and more generally for all the situations where Dutt's method is no longer adequate.
\end{abstract}

Key words: Multiple integral, multivariate normal distribution, independent culling level selection, multivariate probability integrals.

\section{Résumé}

Intérêt en génétique quantitative des méthodes de Dutt et de Deak pour le calcul numérique des intégrales de la loi multinormale

Le calcul numérique d'intégrales de lois multinormales est souvent rendu nécessaire dans les études de génétique quantitative : c'est en particulier le cas pour l'évaluation des effets génétiques d'une sélection à niveaux indépendants sur plusieurs caractères corrélés, pour certaines méthodes d'analyse de caractères discontinus ou pour certaines études de sélection portant sur des effectifs limités.

Les méthodes de Dutt et de Deak peuvent satisfaire une grandè partie des besoins des généticiens. Celles-ci sont présentées dans cet article et leur précision est analysée de façon détaillée. Il apparaît que la méthode de Dutt est remarquablement précise pour les dimensions 1 à 5 , sauf lorsque les seuils de troncature ou les corrélations entre variables sont très élevés en valeur absolue. La méthode de Deak, moins précise, convient mieux pour les dimensions supérieures (de 6 à 20) et d'une manière générale pour toutes les situations où la méthode de Dutt est inadéquate.

Mots clés : Intégrale multiple, distribution multinormale, sélection à niveaux indépendants. 


\section{Introduction}

Usually the continuous traits on which selection is performed are supposed to follow, at least in the base population, a normal distribution. Indeed, the number of genes involved is assumed to be high and the effect of the genetic variations at a given locus is considered to be small (polygenic model). Furthermore, the joint action of environmental effects which are not easily recorded also follows a normal distribution since it supposedly results from many distinct causes, each one with small individual effect.

Discrete traits (fertility traits, calving ease, subjective notes, etc.) cannot be directly described by a normal distribution. However, one possible way to numerically process them is to assume, as did DempSTER \& LERNER (1950), that they are the visible discontinuous expression of an underlying unobservable continuous variable.

Within this general framework, knowledge of the value of normal probability integrals if often required and consequently the scope of corresponding numerical methods is large. Three examples can be mentioned.

1 - Selection procedures deal generally with several traits and selection is often performed not on an overall index combining all traits but through successive stages on one (or more) trait (s) (mainly because information is obtained sequentially and because the cost of selection programs has to be minimized or even because the required economic weights are difficult to define properly).

This situation occurs, for example, in dairy cattle breeding schemes (Ducroce, 1984). After selection on $n$ traits, the evaluation of the average genetic superiority of the selected animals for a given trait (not necessarily one of those on which selection was performed) requires the computation of $n$ integrals of dimension $n-1$ (JAIN \& AмвLE, 1962). It should also be observed that, in practice, the selection procedures are not realized through prespecified thresholds for each trait but through fixed selected proportions of animals at each stage. The derivation of the truncation thresholds given the selected proportions can be done using Newton-Raphson type algorithms involving derivatives which are, once again, (multiple) integrals.

2 - The processing of discrete variables using continuous underlying variables is frequently performed assuming that the corresponding distributions are of logistic or multivariate logistic type (JoHNSON \& KoTz, 1972 ; Bishop et al., 1978). This is due to the similarities they exhibit with the normal or multivariate normal distributions and to the ease of computing their cumulative distributions given the thresholds (logits) or vice versa. The return to strict normality may be desirable in a polygenic context (Gianola \& Foulley, 1983 ; Foulley \& Gianola, 1984) leading to the computation of normal or multivariate normal probability integrals. In practice, with $\mathbf{n}$ discrete variables, each one with $r_{i}$ subclasses ( $i=1$ to $n$ ), the optimum $\sum_{i=1}^{n}\left(r_{i}-1\right)$ thresholds have to be derived (for example using the maximum likelihood method) from the computation of $\left(\prod_{i=1}^{n}\left(r_{i}-1\right)\right)$ different probabilities (which are integrals of dimension $n$ ) and their derivatives.

3 - Selection often involves a limited number of candidates, especially in males (for example in dairy cattle). This, along with the fact that the selected males do not have the same probability to contribute to the procreation of the next generation 
(RoBERTSON, 1961) makes it useful to have a knowledge of the corresponding increase in inbreeding. This last phenomenon is generally not taken into account. Burrows (1984) shows that this problem can be approached using simple and double integrals of normal distributions, provided normality is restored at each generation. In particular, the double integral describes the probability that 2 animals randomly drawn in the same family simultaneously meet the selection criterion.

Despite the importance of the situations where computations of multivariate normal integrals are required in quantitative genetics, it is surprising to notice that geneticists either consider that the problems cannot be correctly solved beyond the dimensions 2 or 3 (SAXTON, 1982; SMITH \& QUAAS, 1982) or use approximations such as, for example, the assumption of preservation of normality for all the variables after truncation selection on several of them (Cunningham, 1975 ; Niebel \& Fewson, 1976 ; Cotterill \& James, 1981 ; Mukai et al., 1985) or even limit the scope of their studies to traits assumed to be uncorrelated.

The only situations where the integrals would be relatively easier to compute seem to be the orthant case, where all the truncation points are zero (KENDALl, 1941 ; Plackett, 1954 ; Gupta, 1963 ; Johnson \& Kotz, 1972) or cases where the correlation matrix has a special structure (DUNnETt \& Sobel, 1955 ; IhM, 1959 ; Curnow, 1962 ; GuPTA, 1963 ; BechHofer \& TAMHANe, 1974 ; Six, 1981 ; El Lozy, 1982). It is obvious that the general needs of geneticists are often quite far from these particular cases.

A review of the literature, which is by no means exhaustive, reveals the availability of 4 general methods that take into account the normality of the distribution :

- Kendall (1941) [Computation of sums of convergent tetrachoric series].

- Milton (1972) [Dimension reduction and repeated Simpson quadratures].

- Dutr (1973, 1975) and Dutr \& Soms (1976) [Computation of a finite sum of Fourier transforms, each one evaluated by GAuss-Hermite quadrature].

- Deak (1976, 1980, 1986) [Computation by Monte-Carlo simulation using special implementations to reduce the sampling variance].

The purpose of this paper is to emphasize the potential of these last 2 methods because they do not seem to be very well known (seldom quoted, at least), even Dutt's method which is more than 10 years old... A further objective is to analyze the precision of these methods more systematically than was done by their authors, our purpose being their use in quantitative genetics through powerful and reliable algorithms.

\section{Methods}

We want to evaluate :

$$
\begin{aligned}
L_{s_{1} \ldots s_{n} ; r_{1} \ldots r_{c}} & =\operatorname{Prob}\left(x_{1}>s_{1}, \ldots, x_{n}>s_{n}\right) \\
& =\int_{s_{1}}^{+\infty} \ldots \int_{s_{n}}^{+\infty} f_{n}\left(x_{1}, \ldots x_{n}\right) d x_{1} \ldots d x_{n}
\end{aligned}
$$

where $f_{n}\left(x_{1}, \ldots x_{n}\right)$ is the joint density of the n-variate normal distribution. $s_{1}, \ldots s_{n}$ are the truncation points of the $n$ standardized variables. $r_{1}, \ldots, r_{c}$ are the correlations among the $c=n(n-1) / 2$ pairs of variables. 


\section{A. Kendall's method}

The probability $\mathbf{L}$ to be computed is the sum of a convergent series involving tetrachoric functions. We have :

$L=\sum_{k_{1}=0}^{\infty} \ldots \sum_{k_{j}=0}^{\infty} \ldots \sum_{k_{c}=0}^{\infty} \prod_{j=1}^{c} \frac{r_{j}^{k_{j}}}{k_{j} !} \prod_{i=1}^{n} \sqrt{\alpha_{i} !} \tau_{\alpha_{j}}\left(s_{i}\right)$

where $i$ is a variable index $(i=1, \ldots n)$

$\mathrm{j}$ is a pair index $(\mathrm{j}=1, \ldots \mathrm{c}$ with $\mathrm{c}=\mathrm{n}(\mathrm{n}-1) / 2)$

$\mathrm{k}_{\mathrm{j}}$ is an expansion index (positive integer from 0 to $+\infty$ ) varying independently for each pair index

$\alpha_{i}=\Sigma k_{j}$ for all pairs which do not include index $i$

$\tau_{\alpha}$ refers to the tetrachoric function of $\mathrm{x}$ of order $\alpha$ :

$\tau_{\alpha}(x)=\frac{1}{\sqrt{\alpha !}} \cdot \frac{1}{\sqrt{2 \pi}} \cdot \exp \left(-x^{2} / 2\right) \cdot H_{\alpha-1}(x)$

and $H_{\alpha}(x)$ is the Hermite polynomial of order $\alpha$, defined by :

$H_{\alpha}(x)=(-1)^{\alpha} \exp \left(x^{2} / 2\right) \cdot \frac{d^{\alpha}\left(\exp \left(-x^{2} / 2\right)\right)}{{d x^{\alpha}}^{\alpha}}$

Without including the computation of factorials, this method roughly requires the computation of $n^{4} k_{M}^{2} / 4$ elementary terms, where $k_{M}$ is the maximum order used in practice in the expansion (the value of $\mathrm{k}_{\mathrm{M}}$ to be used in obtaining a given precision increases with the absolute value of the correlation coefficients). This method was used for example by Burrows (1984) for 2 dimensions. In fact, this method is unfeasible for $n>2$, due to very tedious computations and slow or even non-existent convergence (HARRIS \& Soms, 1980) for intermediate or high values of the correlations $r_{j}$.

\section{B. Milton's method}

A minimum of theory is required in this method since it consists in empirically computing the multiple integral starting from its innermost one. At this stage, the unidimensional normal cumulative distribution is involved and can be computed using one of the numerous polynomial approximations available (PATEL \& READ, 1982). The algorithm actually used is described in Milton \& Hotchkiss (1969). For the following integrals, Simpson's general method is used : the function to be integrated is evaluated at regular intervals and the computed values are summed using very simple weighting factors (AtKinson, 1978 ; Bakhvalov, 1976 ; Mineur, 1966). The accuracy of Simpson's method obviously depends on the interval length. Similarly, to achieve a given precision, the interval length to use can be derived. Shorter intervals are required as lower orders of integration are considered, in order to maintain the overall error at a given value. This leads to large computation times when an absolute error less than $10^{-4}$ is desired and when $n$ is more than 3 (Milton, 1972). Dutr (1973), when comparing the computation times of his method to Milton's, found his to be much faster at a given precision. 


\section{Dutt's method}

This method involves many mathematical concepts. In this section, only the guiding principles are presented, with the main analytical details reported in Appendix 1.

The joint density function of the $n$ normal variables can be expressed using its characteristic function (it is its Fourier transform), which allows the decomposition of the integral into a linear combination of other integrals of equal or lesser dimension than $n$ (GuRLaND, 1948). These integrals have integration limits $(-\infty,+\infty)$ independent of the initial truncation points and therefore can be evaluated using precise numerical integration methods.

The integration range is then shortened to $(0,+\infty)$ using, instead of the function to be integrated, its central difference about 0 . This change permits a reduction, for a given precision, in the number of points at which the function has to be evaluated for the quadrature.

The numerical computation itself is carried out according to Gauss' general method (AtKinson, 1978 ; BaKhValov, 1976 ; Mineur, 1966) : the function to be integrated is evaluated at well defined points (roots of orthogonal polynomials) and the resulting values are summed using weights which are themselves the result of computable integrals... This procedure is less simple than Simpson's but is much more powerful : the function to be integrated is approximated by a polynomial of degree 2 (over a given interval) in Simpson's case, and of degree $2 n^{\prime}-1$ in Gauss' case, where $n^{\prime}$ is the number of roots considered. For these orthogonal polynomials, the quadrature gives an exact result. Here, the functions to be integrated are of the type $\left\{\exp \left(-x^{2} / 2\right) . f(x)\right\}$ and the more convenient polynomial to use for the quadrature is the above mentioned Hermite polynomial. Moreover, since the integration range is $(0,+\infty)$ and the functions $f(x)$ are not defined at $x=0$, only the $n^{\prime}$ positive roots and corresponding weights of the Hermite polynomial of degree $2 n^{\prime}$ are considered.

\section{Deak's method (details in appendix 2)}

Using the Cholesky decomposition of the correlation matrix, it is possible to generate sets of $n$ correlated standardized normal variables from $n$ independent normal variables. The position of these variables with respect to the $n$ truncation points defines an indicator variable for each realization. If we have $\mathrm{N}$ trials with $\mathrm{N}^{*}$ successes, the probability considered is estimated by $\mathrm{N}^{*} / \mathrm{N}$.

Deak's algorithm results from developing this method in such a way as to reduce its sampling variance which is very large otherwise.

- The $\mathrm{n}$ independent normal variables are initially normalized, each normalized vector corresponding to a whole family of colinear vectors. Only some of these vectors, however, fulfill the conditions set up by the truncation points. DEAK demonstrated that knowledge of the normalized vector alone and of an algorithm to compute the cumulative distribution function of a $\chi^{2}$ variable is sufficient to determine a priori the probability of realization over all the corresponding original vectors. This recognition permits a considerable increase in precision for a given number of trials.

- In addition, the original vectors are generated in groups of $\mathrm{n}$ and transformed to an orthonormalized base of dimension $n$ from which $2 n(n-1)$ statistically dependent 
normalized vectors are drawn. On the whole, it is as if $2(n-1)$ families of colinear vectors were associated to each original vector actually drawn, without the need to generate the former.

\section{Results and discussion}

\section{A. Dutt's method}

\section{Precision}

\section{a) General problems}

The error resulting from applying the Gauss quadrature has a theoretically computable upper bound. In the unidimensional case and with $n^{\prime}$ positive roots of the Hermite polynomial of degree $2 n^{\prime}$, the theoretical expressions involve the maximum of the derivative of order $2 n^{\prime}$ of the function to be integrated $f(x)$. This leads to very tedious computations that could, to the limit, be envisioned. However, in the higher dimensional cases, the computation of the derivative is very complex, even for small $\mathbf{n}^{\prime}$, and the determination of its maximum is unfeasible.

Durt (1973) emphasized the precision of his method by comparing the numerical results obtained for the orthant case in 4 dimensions to exact results computable for this particular case. He noted that the precision increased with the number of roots used and with the value of the correlation matrix determinant, the precision being already in the range of $10^{-3}$ for a determinant equal to zero. Hence the situation seemed very favorable. However, DEAK (1980), while pointing out that Dutt's method is the most precise one presently available for numerical computation of lower dimensional $(\leqslant 5)$ integrals, stressed its sensitivity to the value of the determinant. Furthermore, many personal observations have shown that the precision problem seems to have been underestimated by DuTr and that a careless use of this method may lead to obvious errors in certain cases. This justifies a more systematic study of this precision in order to better define the conditions of its reliable use. In particular, it seems essential to look at situations where truncation points are no longer zero and where correlations between traits are not necessarily positive. However, reference results as were available for the orthant case do not exist. Therefore, we will consider only more specific integrals for which quasi exact results can be derived (what is meant by " quasi exact » will be clarified later).

Finally, it must be noted that a less rigorous semi-empirical method to check precision could have been used, as proposed by RALSTON \& WiLf (1967), BaKhVALOV (1976), CoHEN et al. (1977). It consists of comparing the results from computations of integrals using different values of $\mathrm{n}^{\prime}$. Theoretically, an increase in $\mathrm{n}^{\prime}$ should lead to a better precision of the evaluation (approximation by a polynomial of higher degree) as long as cumulated rounding errors do not counterbalance it. This method has not been adopted because the convergence rate for increasing values of $n^{\prime}$ is not really known and computations themselves become too tedious for combinations of large values of $n$ and $\mathrm{n}^{\prime}$.

\section{b) Unidimensional case}

The reference results are those tabulated by WHITE (1970) for which the value of the truncation point corresponding to a given probability is specified at 20 decimal 


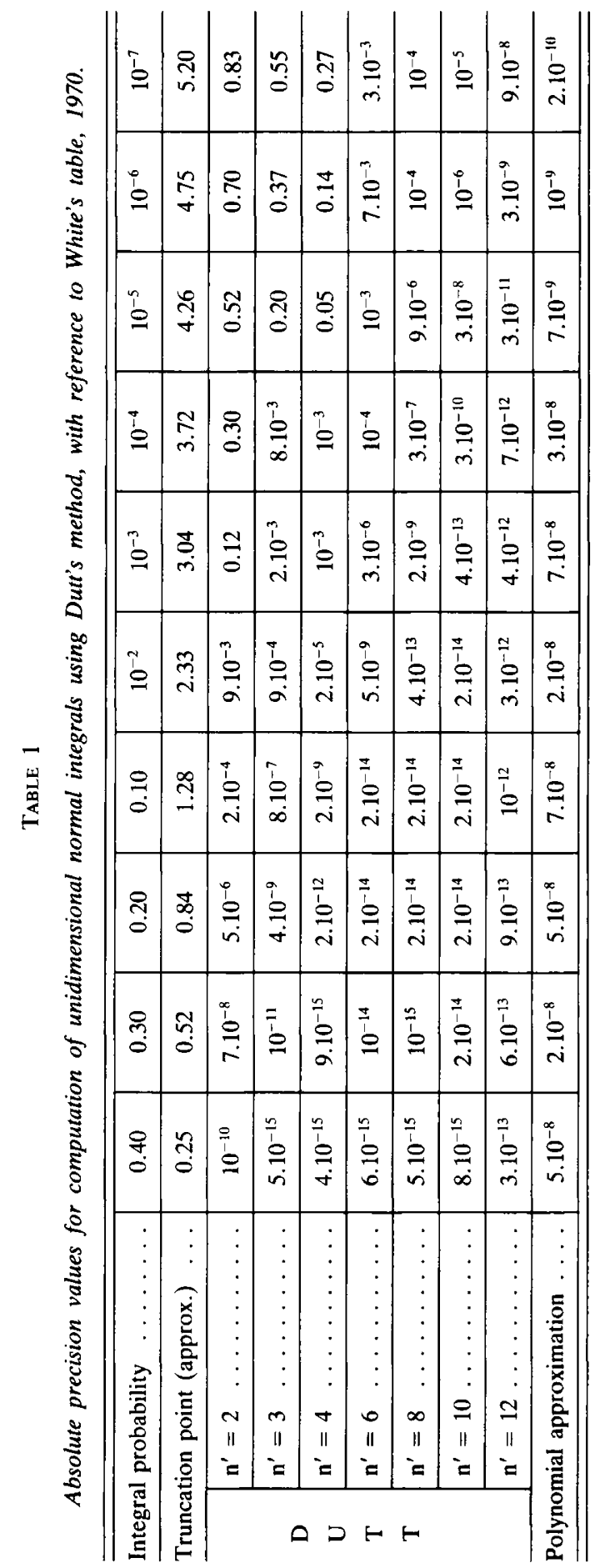


points. In table 1 , the absolute errors when Dutt's method is applied are presented for 10 different truncation points and for 7 values of the number of positive roots $\left(\mathrm{n}^{\prime}\right)$ of the Hermite polynomial (in this table, only the first two decimal points of the corresponding truncation point are shown, but White's 20 decimal points are actually used for the computations).

The probabilities for a value of $\mathbf{n}^{\prime}$ from 2 to 10 were computed using the roots and weighting factors supplied by Abramowitz \& Stegun (1972) for the Hermite polynomials (taking into account, however, that the base function they used was exp $\left(-x^{2}\right)$ and not $\exp \left(-x^{2} / 2\right)$ ). For $n^{\prime}=12$, roots and weights were derived using personal algorithms which yield exactly the same results as Abramowitz \& STEgun for the dimensions they tabulated.

A very clear interaction between truncation points and number of roots can be seen as far as precision is concerned. Dutt's method can be used very accurately in terms of absolute and relative errors by taking 10 positive roots and up to a truncation point of about \pm 4.5 . Our attempt to increase the precision over a wider range gave unsatisfactory results since the improvement for high threshold values was balanced by a slight decline elsewhere (the limit of precision using 8-byte floating point representation is probably reached). In fact, many specialized algorithms for the unidimensional case are available (PATEL \& READ, 1982). Among those, the polynomial approximation referred to as 26.2 .17 by ABramowitz \& STEGUN (1972) and derived by Hastings (1955) is often used because of its simplicity and precision. It is observed that its precision is greater than Dutt's for truncation points larger than 4.5 and therefore was used in such cases.

c) Dimensions 2 to 6

a) Reference algorithm

In the particular case where all correlations are equal and positive, it can be shown that the integration order is always reduced to 2 (DunNeTr \& SoBel, 1955 ; Owen, 1962 ; GUPTA, 1963) :

$\operatorname{Prob}\left(x_{1}<s_{1}, \ldots x_{n}<s_{n}\right)=\int_{-\infty}^{+\infty} \frac{1}{\sqrt{2 \pi}} \exp \left(-z^{2} / 2\right) \prod_{i=1}^{n} F\left(\frac{s_{i}-z \sqrt{r}}{\sqrt{1-r}}\right) d z$

where $\mathrm{F}$ is the cumulative distribution of the unidimensional normal distribution and $r$ is the correlation coefficient between each pair of variables.

Such computations present a more favourable situation than the general case, since they introduce only once both the above mentioned algorithm for the unidimensional case and the Gauss quadrature. This is what we called quasi exact results.

B) Influence of the truncation points

Computation results for absolute precision are shown in table 2 for dimensions 2 to 6 , truncation points of -4 to +4 and step length of 1 . These truncation points are identical for each variable. The correlation value between variables depends on $\mathbf{n}$ and is equal to $1 /(1+\sqrt{n})$; the determinant of the correlation matrix, a supposed factor of variation in precision, thus becomes less sensitive to the value of $n$ (OWEN, 1962).

As indicated by DuTT, the probability estimates for the orthant case, i.e. for all truncation points equal to zero, are indeed very precise (error less than $10^{-5}$ ) for all the 


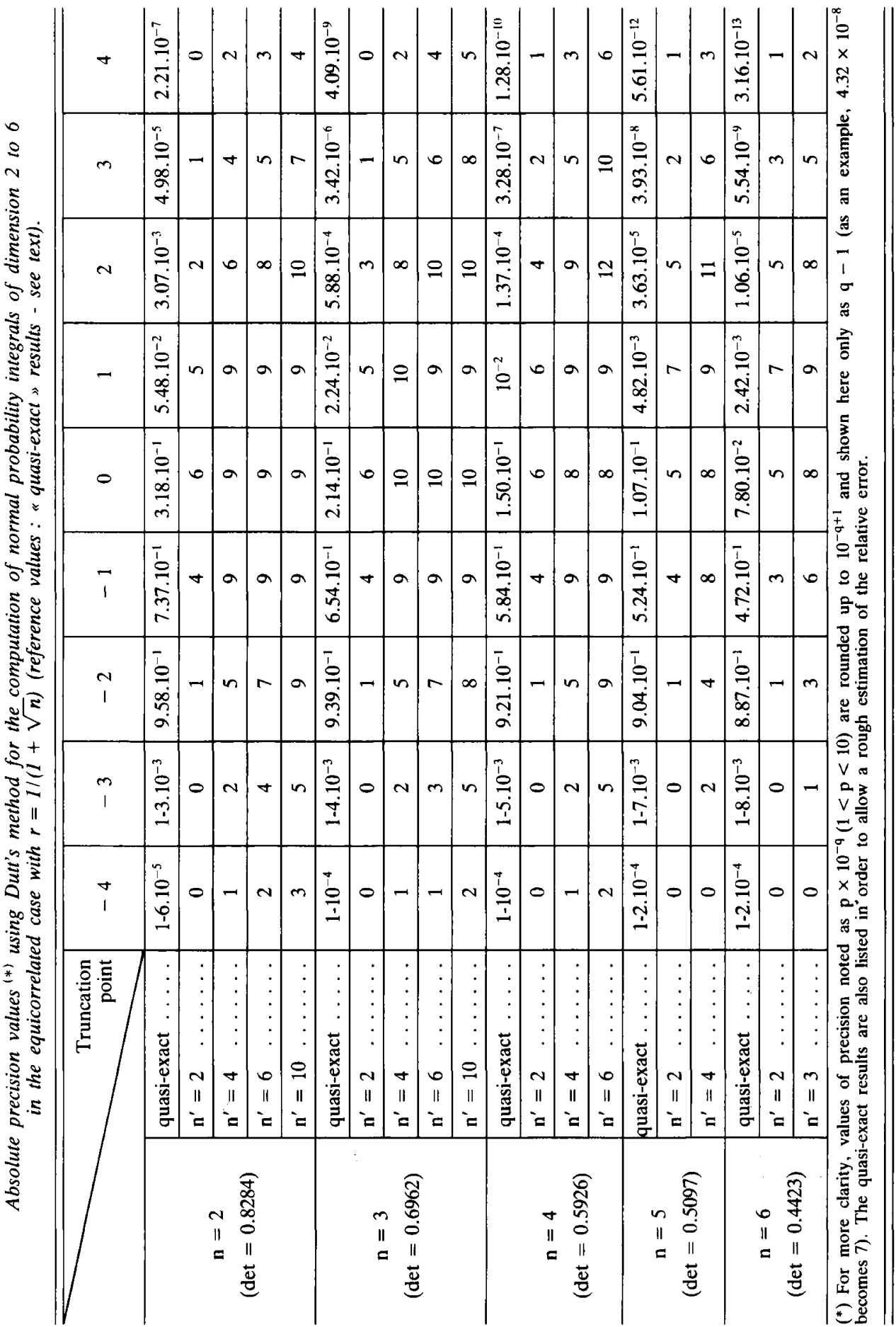


dimensions considered, even with a low number of roots of the Hermite polynomial. In fact, the absolute precision is almost maximum for this category of truncation points. To either side of these central values, the precision decreases in a non-symmetrical fashion. For very large positive truncation points (3 to 4), absolute precision is much larger than for corresponding negative ones, whereas the contrary is true for relative precision. The use of a large number of roots, when possible, extends the range of reliable use of the algorithm. With 6 to 10 roots, the absolute precision can be considered satisfactory (less than $10^{-5}$ ), for dimensions 2 to 4 and truncation points -3 to +3 . However, for very low values of the probability, the relative error can become as high as $10^{-1}$. For dimensions 5 and 6 , the possible number of roots is lower (3 or 4) due to computation complexity, and the range of reliable use is narrower $(-2$ to +2$)$.

$\gamma)$ Influence of the correlation coefficients

We will only consider here correlation coefficients having on the average larger absolute values than in the previous test. However, to permit computation of reference results for more than 2 dimensions, we must restrict our study to particular situations. For 4 dimensions, we will assume that the 4 variables are separated into 2 mutually independent blocks of 2 variables.

Tables 3 and 4 respectively outline the results obtained for 2 and 4 dimensions when absolute values of non-zero correlation coefficients are $0.5,0.7$ or 0.9 . The previous section's conclusions for 2 dimensions are applicable here with the exception of very large correlation coefficients (of about \pm 0.9 ) for which a noticeable drop in precision is seen. The results of table 4 confirm this fact : only one correlation coefficient with a large absolute value is sufficient to considerably decrease precision. The sign of this coefficient has only a small effect on the absolute precision but this is obviously no longer the case when relative precision is considered since integrals involving negatively correlated variables have a smaller value and are therefore more poorly estimated in relative value.

It can be noted that the unfavorable effect of several large coefficients on absolute precision is not cumulative. This suggests that it is not the value of the determinant which limits precision but rather the largest absolute value of the correlation coefficient. Indeed, for a same determinant the precision is generally greater in the equicorrelated case (last row in table 4) than when some of the correlations are very high (first row of table 4). In fact, in the general case, this limiting factor could be the smallest eigenvalue of the correlation matrix, but it was not possible to prove it without additional reference results.

\section{Computation times}

Dutt's method involves the computation of "elementary " expressions which are the product of an exponential and a trigonometric function. The number of these expressions increases very quickly with $n^{\prime}$, the number of positive roots of the Hermite polynomial used, since it is equal to :

$$
\sum_{i=1}^{n}\left(\begin{array}{l}
n \\
i
\end{array}\right) 2^{i-1} \cdot n^{\prime i}
$$




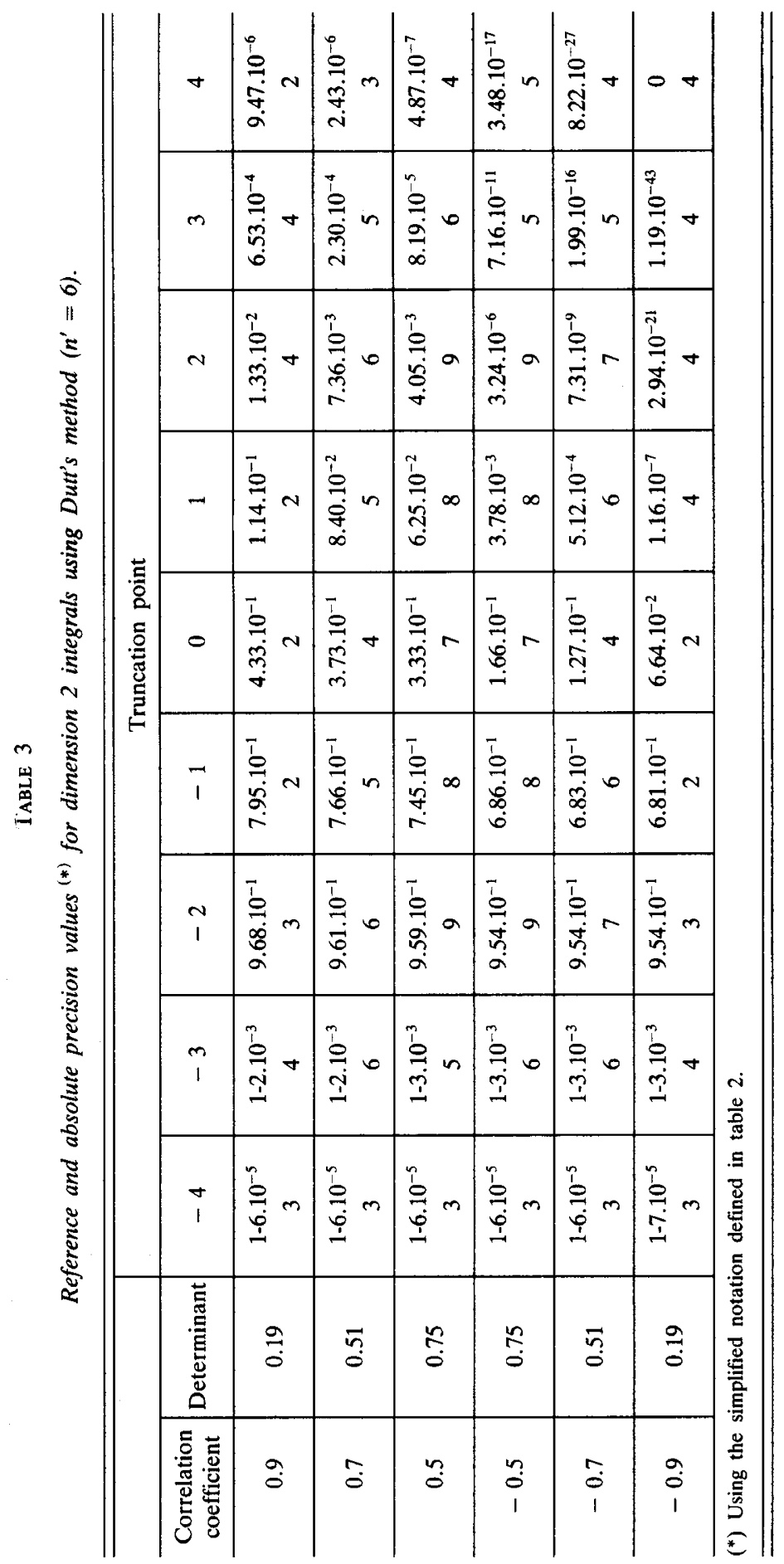


TABLE 4

Absolute precision values ${ }^{(*)}$ of Dutt's method for integrals of dimension 4 $\left(r_{13}=r_{14}=r_{23}=r_{24}=0\right)$.

\begin{tabular}{|c|c|c|c|c|c|c|c|c|c|c|c|}
\hline \multirow[b]{2}{*}{$r_{12}$} & \multirow[b]{2}{*}{$\mathbf{r}_{34}$} & \multirow[b]{2}{*}{ determinant } & \multicolumn{9}{|c|}{ Truncation point } \\
\hline & & & -4 & -3 & -2 & -1 & $\mathbf{0}$ & 1 & 2 & 3 & 4 \\
\hline \multirow[t]{6}{*}{0.9} & 0.9 & 0.0361 & 3 & 3 & 3 & 2 & 2 & 3 & 5 & 6 & 5 \\
\hline & 0.7 & 0.0969 & 3 & 4 & 3 & 2 & 2 & 3 & 6 & 7 & 6 \\
\hline & 0.5 & 0.1425 & 3 & 4 & 3 & 2 & 2 & 3 & 6 & 8 & 7 \\
\hline & -0.5 & 0.1425 & 3 & 4 & 3 & 2 & 3 & 5 & 9 & 8 & 7 \\
\hline & -0.7 & 0.0969 & 3 & 4 & 3 & 2 & 3 & 5 & 9 & 8 & 7 \\
\hline & -0.9 & 0.0361 & 3 & 4 & 3 & 2 & 2 & 5 & 5 & 7 & 7 \\
\hline \multirow[t]{5}{*}{0.7} & 0.7 & 0.2601 & 2 & 5 & 6 & 4 & 4 & 5 & 8 & 8 & 7 \\
\hline & 0.5 & 0.3825 & 2 & 5 & 6 & 5 & 5 & 6 & 8 & 9 & 7 \\
\hline & -0.5 & 0.3825 & 2 & 5 & 6 & 5 & 5 & 7 & 11 & 9 & 8 \\
\hline & -0.7 & 0.2601 & 2 & 6 & 6 & 5 & 5 & 7 & 9 & 9 & 8 \\
\hline & -0.9 & 0.0969 & 2 & 4 & 3 & 2 & 2 & 5 & 6 & 7 & 7 \\
\hline \multirow[t]{4}{*}{0.5} & 0.5 & 0.5625 & 2 & 5 & 9 & 7 & 8 & 9 & 11 & 10 & 8 \\
\hline & $-0,5$ & 0.5625 & 2 & 5 & 9 & 8 & 8 & 9 & 11 & 9 & 9 \\
\hline & -0.7 & 0.3825 & 2 & 5 & 7 & 6 & 5 & 7 & 9 & 9 & 8 \\
\hline & -0.9 & 0.1425 & 2 & 4 & 3 & 2 & 2 & 5 & 6 & 8 & 8 \\
\hline \multirow[t]{3}{*}{-0.5} & -0.5 & 0.5625 & 2 & 5 & 8 & 8 & 8 & 10 & 14 & 11 & 10 \\
\hline & -0.7 & 0.3825 & 2 & 6 & 7 & 6 & 5 & 8 & 12 & 11 & 9 \\
\hline & -0.9 & 0.1425 & 2 & 4 & 3 & 2 & 3 & 6 & 9 & 9 & 9 \\
\hline \multirow[t]{2}{*}{-0.7} & -0.7 & 0.2601 & 2 & 5 & 6 & 5 & 5 & 9 & 15 & 10 & 9 \\
\hline & -0.9 & 0.0969 & 2 & 4 & 3 & 2 & 3 & 7 & 11 & 9 & 8 \\
\hline-0.9 & -0.9 & 0.0361 & 2 & 3 & 3 & 2 & 3 & 8 & 8 & 8 & 8 \\
\hline \multicolumn{2}{|c|}{$r_{12}=r_{13}=\ldots=r_{34}=0.78$} & 0.0361 & 4 & 4 & 3 & 3 & 3 & 4 & 3 & 5 & 4 \\
\hline
\end{tabular}

$\left(^{*}\right)$ Using the simplified notation defined in table 2 .

As an example, some recorded computation times are presented in table 5. These times are only indicative since we used an advanced - and moreover interpreted language (APL) but with the possibility when the memory size allows it (here 2 Megabytes maximum) to partly compensate this handicap by using vectorial methods when several independent integrals are to be evaluated at the same time. In addition, we cannot pretend to have written optimal programs. 
TABLE 5

Computation times ${ }^{(*)}$ for multivariate normal probability integrals using Dutt's method (in CPU time, on an IBM 3031 computer).

\begin{tabular}{|c|c|c|c|c|c|}
\hline Dimension & $\begin{array}{l}\text { Number } \\
\text { of roots }\end{array}$ & Time & Dimension & $\begin{array}{l}\text { Number } \\
\text { of roots }\end{array}$ & Time \\
\hline 1 & 10 & $14 \mathrm{~ms}(2 \mathrm{~ms})$ & \multirow{2}{*}{4} & \multirow{2}{*}{$\begin{array}{l}2 \\
4 \\
6\end{array}$} & \multirow{2}{*}{$\begin{array}{rr}0.6 \mathrm{~s} \\
2.7 \mathrm{~s} \\
11 & \mathrm{~s}\end{array}$} \\
\hline \multirow[b]{2}{*}{2} & \multirow{2}{*}{$\begin{array}{r}2 \\
4 \\
6 \\
10\end{array}$} & \multirow{2}{*}{$\begin{array}{l}27 \mathrm{~ms}(7 \mathrm{~ms}) \\
36 \mathrm{~ms}(17 \mathrm{~ms}) \\
50 \mathrm{~ms} \\
95 \mathrm{~ms}\end{array}$} & & & \\
\hline & & & 5 & $\begin{array}{l}2 \\
4\end{array}$ & $\begin{array}{r}1.7 \mathrm{~s} \\
24 \mathrm{~s} \\
\end{array}$ \\
\hline \multirow{2}{*}{3} & \multirow{2}{*}{$\begin{array}{r}2 \\
4 \\
6 \\
10\end{array}$} & \multirow{2}{*}{$\begin{aligned} & 76 \mathrm{~ms}(41 \mathrm{~ms}) \\
& 238 \mathrm{~ms}(87 \mathrm{~ms}) \\
& 642 \mathrm{~ms} \\
& 2200 \mathrm{~ms}\end{aligned}$} & 6 & $\begin{array}{l}2 \\
3\end{array}$ & $\begin{array}{r}7.3 \mathrm{~s} \\
52 \mathrm{~s}\end{array}$ \\
\hline & & & 7 & $\begin{array}{l}2 \\
3\end{array}$ & $\begin{array}{l}27 \mathrm{~s} \\
5 \mathrm{mn}\end{array}$ \\
\hline
\end{tabular}

$\left(^{*}\right)$ Values between parentheses were obtained using vector or matrix arguments.

It should be observed that the computation times required for a reliable use of the method (i.e. the number $n^{\prime}$ of roots being at least 4 or 6) become large when $n$ is equal to 5 . For $n=6$ to 7 , computation times are extremely large, even when a small number of roots is used.

\section{B. Deak's method}

\section{General characteristics}

The method described is unbiased and does not present any particular problem with respect to the values of the truncation points. Moreover, it is insensitive to the nature of the relationships between variables owing to usage of the Cholesky decomposition. However, the method does not tolerate any error leading to negative eigenvalues in the construction of the correlation matrix. This security does not exist with Dutt's method where negative values or values larger than 1 for probabilities may be obtained in such cases.

It also becomes possible to deal with large values of $\mathbf{n}$; effectively DEAK computed probabilities with $\mathrm{n}$ up to 50 . According to the author, this is the main justification of the method.

\section{Numerical investigations}

a) Unbiasedness

Deak (1976) showed that the method he proposed is unbiased : he observed a (slow) convergence of the computed probabilities toward the true value of the corresponding integral, in cases for which this value could be computed a priori. The results presented in table 6 , for 4 dimensions and with 2 different correlation matrices - the one used in table 2 and one of those used in table 3 - empirically support this assertion (we limited ourselves to these examples because computations were quite tedious). 


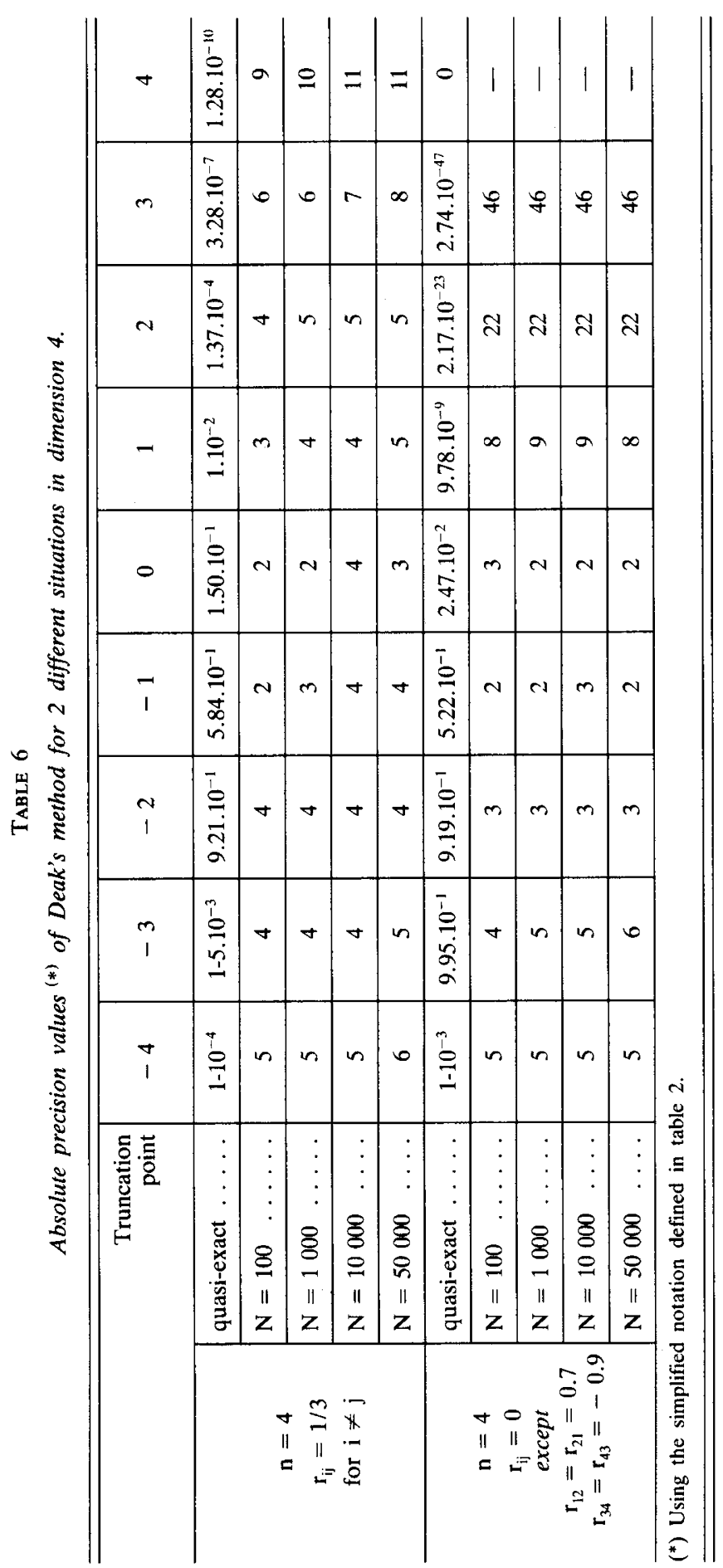




\section{b) Precision}

The major difficulty is encountered in evaluating a priori the sampling variance, to characterize the domain where this method can be applied, and to compare it with Dutt's method. The theoretical expression of this variance is not given by the author.

However, to get an approximation a posteriori of the precision of the computed probability, it is always possible to use the observed variance of the $\mathrm{N}$ independent evaluations which are averaged to obtain the final result (see appendix 2).

To identify the factors influencing $\sigma^{2}(\hat{\mathbf{p}})$, computations were limited to dimensions $\mathrm{n}=4$ to 10 , for which computation times are reasonable. To the 7 situations studied by DEAK (1980), we added 90 new examples (20 for $n=4$ or 5,10 for $n=6$ to 10). Each situation corresponds to a random drawing of truncation points in the interval $[-4$, +4 ]. Positive definite correlation matrices were randomly generated using the method of Bendel \& Mickey (1978). For each integral, $N=1000$ independent evaluations were performed to improve our estimate of the sampling variance.

By analogy with the binomial distribution, let $v=[p(1-p) / f(n)]$ be the form of the sampling variance of an elementary evaluation of the integral, and assume the approximation $\mathrm{p}(1-\mathrm{p}) \approx \hat{\mathrm{p}}(1-\hat{\mathrm{p}})$ holds. By regression, we found that a polynomial approximation of $f(n)$ is given by $n(n+100)$. After doubling the value of $v$, an upper bound of the "true " sampling variance was always obtained. Then, the sampling variance when the $\mathrm{N}$ independent estimates are averaged is :

$$
\sigma^{2}(\hat{\mathrm{p}})<\frac{2 \hat{\mathrm{p}}(1-\hat{\mathrm{p}})}{\operatorname{Nn}(\mathrm{n}+100)}
$$

Notice that $\sigma^{2}(\hat{p})$ is smaller for large $n$ since the number of orthonormal vectors which are used is much larger, as explained in appendix 2.

Assuming that $\hat{\mathrm{p}}$ roughly follows a normal distribution and that the maximum absolute error is $3 \sigma(\hat{\mathrm{p}})$, the upper bound for this absolute error $\epsilon$ is :

$$
3 \sqrt{\frac{2 \hat{p}(1-\hat{p})}{N n(n+100)}}
$$

This prediction is verified by the results presented in table $6-$ which were not used to derive this upper bound. In practice, if $N=100$ is taken as suggested by Deak, $\epsilon<10^{-2}$ is obtained for all probabilities $p$ and sometimes $\epsilon<10^{-3}$, in particular when $\mathrm{p}$ is close to 1 .

A notable increase in precision cannot be achieved without increasing considerably the number of trials, as shown in table 6. This indicates that Deak's method, on the average, is not very precise. However, it allows one to get an approximate answer to problems which could not be solved using Dutt's method ( $>5$ or 6 ) with reasonable computation times. It is also a useful complement to Dutt's method when the correlation matrix is very ill-conditioned (example : second situation of table 6).

\section{c) Computation times}

Box-Muller's method (PATEl \& Read, 1982) which is used to generate the variables, necessitates about $0.5 \mathrm{msec}$ on the average for each variable. The uniform random 
variable generator required by this method is the multiplicative congruential generator integrated to the APL language. This generator is identical to the IBM RANDU generator. As shown by FishmAN \& Moore (1982), its properties of independence and uniformity are not excellent. However, to limit computation times, we used it because it was already coded in machine language. The algorithm for the computation of the cumulative $\chi^{2}$ distribution is the one referred to as 26.4 .5 by Abramowitz \& STEGUN (1972), which is suitable for even dimensions and requires the computation of a finite number of terms. The extension to even dimensions when considering an odd number of variables is achieved by adding a dummy variable. Using this method, the computation is very quiek $(0.5 \mathrm{msec}$ for $\mathrm{n}=5$ or $6 ; 0.7 \mathrm{msec}$ for $\mathrm{n}=9$ or $10 ; 1.5 \mathrm{msec}$ for $\mathbf{n}=19$ or 20 ). Incidentally, the constitution of groups of orthonormalized vectors was performed using the Gram-Schmidt method.

Under these conditions, the computation times are $9 \mathrm{sec}$ for $\mathrm{n}=5,40 \mathrm{sec}$ for $\mathrm{n}=10$ and $5 \mathrm{mn}$ for $\mathrm{n}=20$. If a maximal absolute error of about $10^{-2}$ to $10^{-3}$ is acceptable, Deak's method becomes more useful than Dutt's as soon as $n>5$.

\section{Examples of application}

\section{Computation of probabilities involved in multistage selection schemes :}

a) $n \leqslant 5$

The method of choice is Dutt's, except in extreme cases (very high correlations and/or very high absolute values of truncation points). In case of mistrust, we propose to perform the same computation using Deak's method and to compare the differences between the 2 results with the standard error of Deak's estimate. If the difference is too large, Deak's result is prefered. A simple example will illustrate this rule.

Consider a population of cows with 5 recorded lactations $\left(h^{2}=0.25, r=0.5\right.$, genetic correlation between lactations $=1$ ). We would like to determine which fraction of these cows had all their successive average milk yields above a given threshold, after 3,4 or 5 lactations. Successive average milk yields are very highly correlated variables : the correlation between the average of the first $i$ lactations and the average of the first j lactations $(i<j)$ is given by :

$$
\sqrt{\frac{i}{j} \cdot \frac{1+(j-1) r}{1+(i-1) r}}
$$

Where $i=1$ and $j=2, r_{i j}$ is already equal to 0.867 . With $i=4$ and $j=5$, we have $r_{i j}=0.98$. If the threshold is equal to 2 on the standard normal scale, the frequencies we are looking for are equal to $9.0 \times 10^{-3}, 7.6 \times 10^{-3}$ and $6.7 \times 10^{-3}$ at the end of the third, fourth and fifth lactations. The difference between the 2 methods is very small. When the truncation point is equal to 3 , the corresponding frequencies computed using Dutt's method are $3.4 \times 10^{-4}, 2.7 \times 10^{-4}$ and $6.3 \times 10^{-4}$ when this last value should be smaller than the second one! The first computation performed using Deak's method gave the following values: $3.5 \times 10^{-4}, 2.7 \times 10^{-4}$ and $2.5 \times 10^{-4}\left(\sigma_{\hat{p}}=8.4 \times 10^{-5}\right)$. This last result is significantly different from $6.3 \times 10^{-4}$ and is also a more logical one.

b) $n>5$

This is the main domain of application of Deak's method. The availability of such a method is useful, for example, in the study of the genetic structure of a population subject to selection. As an example, the computation of the probability that 2 animals 
selected through independent culling levels on $n$ traits are progeny of the same sire involves integrals of dimension $2 \mathbf{n}$.

2. Optimum truncation points for independent culling level selection involving 3 traits

a) Solution using Dutt's method:

Let us consider an independent culling level selection on 3 correlated traits $X_{1}, X_{2}$, $\mathrm{X}_{3}$ where only the overall selected fraction $\alpha$ is a priori fixed and is the result of 3 successive selections on $X_{1}\left(\alpha_{1}\right), X_{2}\left(\alpha_{2}\right)$ and $X_{3}\left(\alpha_{3}\right)$. We want to derive the combination of selected fractions $\alpha_{1}, \alpha_{2}, \alpha_{3}$ given $\alpha=\alpha_{1} . \alpha_{2} . \alpha_{3}$, such that the expected value of $H=\sum_{i=1}^{3} m_{i} \cdot X_{i}$ for the selected animals is maximized. The $m_{1}$ 's are the economic weights of the 3 traits.

In other words, we want to compute the truncation points $k_{1}, k_{2}, k_{3}$ such that :

$$
L\left(k_{1}, k_{2}, k_{3}\right)=\int_{k_{1}}^{\infty} \int_{k_{2}}^{\infty} \int_{k_{3}}^{\infty} f_{3}\left(x_{1}, x_{2}, x_{3}\right) \cdot d x_{1} \cdot d x_{2} \cdot d x_{3}=\alpha
$$

maximizing :

$$
E\left(H \mid x_{1}>k_{1}, x_{2}>k_{2}, x_{3}>k_{3}\right)=\frac{\sum_{i=1}^{3} M_{i} \cdot z_{i} \cdot J_{i}}{\alpha}\left(J_{\text {AIN } \& \text { AMBLE, 1962) }}\right.
$$

where :

$f_{3}$ is the density function of a trivariate normal distribution.

$$
M_{i}=\sum_{i=1}^{3} m_{i} \cdot r_{i j}
$$

$r_{i j}$ is the correlation coefficient between $X_{i}$ and $X_{j}$

$z_{i}$ is the ordinate of the normal density function at $k_{i}$

$$
\begin{aligned}
& J_{i}=\int_{h_{i j}}^{\infty} \int_{h_{i j}}^{\infty} f_{2, i}\left(t_{1}, t_{2}\right) d t_{1} \cdot d t_{2} \\
& h_{i j}=\frac{k_{j}-r_{i j} k_{i}}{\sqrt{1-r_{i j}^{2}}} j \neq i \quad j^{\prime} \neq i \quad j^{\prime} \neq j
\end{aligned}
$$

$f_{2,1}$ is the density function of a bivariate normal distribution with correlation coefficient equal to the one between $X_{j}$ and $X_{j^{\prime}}\left(j, j^{\prime} \neq i\right)$ given $X_{i}$.

SMITH \& QUAAS (1982) showed that this problem can be solved by equating to zero the partial derivatives of :

$$
\left(\sum_{i=1}^{3} M_{i} \cdot z_{i} \cdot J_{i}\right)-\lambda \cdot\left(L\left(k_{1}, k_{2}, k_{3}\right)-\alpha\right)
$$

with respect to $k_{1}, k_{2}, k_{3}$ and $\lambda$ ( $\lambda$ is a Lagrange multiplier). After eliminating $\lambda$, this leads to a system of 3 equations in $k_{1}, k_{2}, k_{3}$ which is solved iteratively using Newton's method. Normal probability integrals of dimension 1,2 and 3 are involved at each iteration. Table 7 presents the optimum $k_{i}^{\prime}$ 's and $\alpha_{i}^{\prime}$ s for 3 traits such that 
$\mathrm{r}_{12}=\mathrm{r}_{13}=-0.40, \mathrm{r}_{23}=0.25$ with $\mathrm{m}_{1}=1, \mathrm{~m}_{2}=1.1$ and $\mathrm{m}_{3}=1.2$. The stopping criteria are $\left(A_{1}^{2}+A_{2}^{2}+A_{3}^{2}\right)^{0.5}<10^{-4}$, where $A_{i}$ is the $i^{\text {th }}$ left hand side of the system of equations, and $\left|L\left(k_{1}, k_{2}, k_{3}\right)-\alpha\right|<2.10^{-5}$. The corresponding genetic gain for $H$ is compared to what would have been obtained with index selection of same intensity.

TABLE 7

Optimum truncation points for independent culling level selection on 3 correlated traits. (correlations : $r_{12}=r_{13}=-0.40 ; r_{23}=0.25$; weighting factors of the selection index: $m_{1}=1 ; m_{2}=1.1 ; m_{3}=1.2$ ).

\begin{tabular}{|c|c|c|c|c|c|c|}
\hline \multirow{2}{*}{$\begin{array}{l}\text { Overall } \\
\text { selected } \\
\text { proportion }\end{array}$} & \multirow[t]{2}{*}{$\begin{array}{l}\text { Number of } \\
\text { iterations }\end{array}$} & \multicolumn{3}{|c|}{$\begin{array}{l}\text { Truncation points (first line) } \\
\text { and selected proportion (second line) } \\
\text { (in \%) at each stage }\end{array}$} & \multirow{2}{*}{$\begin{array}{c}E\left(H \mid k_{1}\right. \\
\left.k_{2}, k_{3}\right) \\
\text { (in st. dev. } \\
\text { of } H \text { ) }\end{array}$} & \multirow{2}{*}{$\begin{array}{c}\text { Efficiency }= \\
\left(\begin{array}{c}\text { ind. culling } \\
\text { level } \\
\begin{array}{c}\text { index } \\
\text { selection }\end{array}\end{array}\right.\end{array}$} \\
\hline & & 1 & 2 & 3 & & \\
\hline 0.50 & 5 & $\begin{array}{l}-\infty \\
100\end{array}$ & $\begin{array}{c}-0.5815 \\
72.0\end{array}$ & $\begin{array}{c}-0.3854 \\
69.5\end{array}$ & 0.9538 & 0.761 \\
\hline 0.25 & 5 & $\begin{array}{c}-1.7185 \\
95.7\end{array}$ & $\begin{array}{c}-0.0723 \\
51.5\end{array}$ & $\begin{array}{c}-0.1214 \\
50.7\end{array}$ & 1.5641 & 0.783 \\
\hline 0.10 & 5 & $\begin{array}{c}-1.2891 \\
90.1\end{array}$ & $\begin{array}{l}0.3571 \\
32.9\end{array}$ & $\begin{array}{l}0.5513 \\
33.7\end{array}$ & 2.2365 & 0.811 \\
\hline 0.01 & 5 & $\begin{array}{c}-0.8604 \\
80.5\end{array}$ & $\begin{array}{l}1.0734 \\
10.5\end{array}$ & $\begin{array}{l}1.2830 \\
11.8\end{array}$ & 3.5866 & 0.857 \\
\hline 0.001 & 7 & $\begin{array}{c}-0.6539 \\
74.3\end{array}$ & $\begin{array}{c}1.5880 \\
3.3\end{array}$ & $\begin{array}{c}1.8169 \\
4.1\end{array}$ & 4.6686 & 0.883 \\
\hline
\end{tabular}

Classical results described by many authors considering independent culling level selection on 2 correlated traits or several uncorrelated traits are also found here : direct index selection is always more efficient than independent culling level selection but this superiority decreases when the overall selection intensity increases (HAZEL \& LUSH, 1942 ; Young, 1961 ; FinNeY, 1962).

Also, for small selection intensity or when weighting factors are very different, the optimal selection may lead to no selection on one of the traits (Young, 1961; Namkoong, 1970 ; Smith \& QuaAs, 1982 ; Tibau i Font \& Ollivier, 1984).

\section{b) Solution using Deak's method}

Since this method is based on random number generation, the results obtained cannot be reproduced, at least in a practical way. This characteristic and the relatively low precision of Deak's method complicate its use in complex iterative algorithms where convergence is desired. This clearly appears when we try to solve the previous problem using Deak's method to compute the integrals of dimension 2 and 3 - though this does not correspond to its " usual » domain of application.

For small values of the elementary probability integrals $(p<0.03)$, the random fluctuations of the evaluation of these integrals are of the same order of magnitude as their value $p$ and the optimization problem cannot be solved. For large values of $p$, 
Deak's method gives results which slightly vary around the «true values » $( \pm 0.01$ for the truncation points and \pm 0.005 for the probabilities), usually after the same number of iterations as Dutt's method. In some intermediate cases ( $p$ between 0.03 and 0.05 and $p>0.8)$, convergence is sometimes not obtained and it is then necessary to restart the computations. All these facts show that specific problems would arise when using Deak's method within iterative procedures for higher values of $\mathrm{n}$, those corresponding to its actual domain of application (however, it should be kept in mind that this method allows one to consider other problems which would remain without any solution otherwise).

\title{
IV. Conclusion
}

To summarize, an optimal use of Dutt's and Deak's methods can be recommended according to the following general pattern :

Dutt's algorithm can be used for the dimensions 2 to 4 , except when one of the truncation points is out of the interval $(-3,+3)$ or when the smallest eigenvalue of the correlation matrix is inferior to 0.20 . For all these dimensions, the absolute error is at most $10^{-2}$ when 4 roots are used and $10^{-3}$ when 6 roots are used. In fact, the absolute error decreases very quickly when the truncation points become closer to the origin. Indeed, the corresponding values are $10^{-5}$ and $10^{-6}$ in the interval $(-2,+2)$. Therefore, the method is remarkably precise on the average.

For 5 dimensions, the preservation of the same precision becomes difficult for large values of the truncation points ( 2 to 3 ) since the number of roots which can be used consistently with reasonable computation times is more limited (4 in our programs). Thus it may be more advisable to consider the use of Deak's method in such cases.

When Dutt's method is no longer adequate (large truncation points, ill-conditioned correlation matrices or dimension larger than 5), one can resort to Deak's method. The absolute precision is then about $10^{-2}$. However, the stochastic nature of the computation must be taken into account, especially when the corresponding probabilities are involved in iterative algorithms requiring convergence.

Generally speaking, these 2 methods allow one to approach the study of relatively complex genetic problems with good conditions of precision. The short example presented in part III-C-2 is significant in this sense. It becomes possible to easily use algorithms like the one proposed by SMIrH \& QUAAS (1982), which was considered only theoretically by these authors for more than 2 traits because routines for the evaluation of the multidimensional integrals were required to numerically solve the problem.

\author{
Received October 31, 1985. \\ Accepted June 6, 1986.
}

\section{Acknowledgments}

The authors thank an anonymous referee for useful comments. Dr. Deak (Hungarian Academy of Sciences, Budapest) is heartily thanked for providing a yet unpublished paper and giving valuable criticism on their manuscript. The authors are indebted to Miss M.P. LEFrançors (Cornell University) for her help in the translation from french and J.W. JAMES for final linguistic correction. 


\section{References}

Abramowitz M., Stegun I.A., 1972. Handbook of mathematical functions. 1043 pp., National Bureau of Standards, Washington.

AtKinson K.E., 1978. An introduction to numerical analysis. 587 pp., John Wiley, New York.

Bakhvalov N., 1976. Méthodes Numériques. 606 pp., Edition Mir, Moscou.

BechHofer R.E., TAMHANE A.C., 1974. An iterated integral representation for a multivariate normal integral having block covariance structure. Biometrika, 61, 615-619.

Bendel R.B., Mickey M.R., 1978. Population correlation matrices for sampling experiments. Commun. Stat., B7, 163-182.

Bishop Y.M.M., Fienberg S.E., Holland P.W., 1978. Discrete Multivariate Analysis : theory and practice. 577 pp., The M.I.T. press, Cambridge.

Bоотн A.D., 1957. Numerical methods. 195 pp., Butterworths Scientific Public, London.

Burrows P.M., 1984. Inbreeding under selection from unrelated families. Biometrics, 40, 357-366.

Cohen A.M., Cutts J.F., Fielder R., Jones D.E., Ribbans J., Stuart E., 1977. Analysis Numérico (translation of Numerical Analysis, 1977. 366 pp., Mc Graw-Hill book company, Maidenhead), Reverté, Barcelona.

Cotterill P.P., JAmes J.W., 1981. Optimizing two-stage independent culling selection in tree and animal breeding. Theor. Appl. Gen., 59, 67-72.

Cunningham E.P., 1975. Multistage index selection. Theor. Appl. Gen., 46, 55-61.

Curnow R.N., 1962. The numerical evaluation of certain multivariate normal integrals. Ann. Math. Stat., 33, 571-579.

DEAK I., 1976. A többdimenziós tér halmazai valószinüségeinek kiszámitása normális eloszlás esetén. Alkalm. Mat. Lapok, 2, 17-26.

DeAK I., 1980. Three digit accurate multiple normal probabilities. Numer. Math., 35, 369-380.

DEAK I., 1986. Computing probabilities of rectangles in case of multinormal distribution. To be published in J. Stat. Comput. and Simul.

Dempster E.R., LeRner I.M., 1950. Heritability of threshold characters. Genetics, 35, 212-235.

Ducroce V., 1984. Conséquences sur le progrès génétique laitier d'une sélection sur des caractères secondaires chez les bovins. Génét. Sél. Evol., 16, 467-490.

DunNetr C.W., SoBel M., 1955. Approximations to the probability integral and certain percentage points of multivariate analogue of Student's t-distribution. Biometrika, 42, 258-260.

DuTT J.E., 1973. A representation of multivariate probability integrals by integral transforms. Biometrika, 60, 637-645.

DutT J.E., 1975. On computing the probability integral of a general multivariate t. Biometrika, 62, 201-205.

Dutr J.E., Soms A.P., 1976. An integral representation technique for calculating general multivariate probabilities with an application to multivariate $\chi^{2}$. Commun. Stat., 5, 377-388.

EL Lozy M., 1982. Simple computation of a bivariate normal integral arising from a problem of misclassification with applications to the diagnosis of hypertension. Commun. Stat., 11, 21952205.

FinNey D.J., 1962. Cumulants of truncated multinormal distribution. J.R. Stat. Soc., B24, 535-536.

Fishman G.S., MooRe L.R., 1982. A statistical evaluation of multiplicative congruential random number generator with modulus $2^{31}-1$. J. Am. Stat. Ass., 77, 129-136.

Foulley J.L., Gianola D., 1984. Estimation of genetic merit from bivariate "all or none " response. Génét. Sél. Evol., 16, 285-306.

Gianola D., Foulley J.L., 1983. Sire evaluation for ordered categorical data with a threshold model. Génét. Sél. Evol., 15, 201-223.

GuPTA S.S., 1963. Probability integrals of multivariate normal and multivariate t. Ann. Math. Stat., 34, $792-828$.

GurLand J., 1948. Inversion formulae for the distribution of ratios. Ann. Math. Stat., 19, 228-237. 
Harris B., Soms A.P., 1980. The use of the tetrachoric series for evaluating multivariate normal probabilities. J. Multivariate Anal., 10, 252-267.

Hastings C., 1955. Approximations for digital computers. 351 pp., Princeton University Press, Princeton.

Hazel L.N., LuSh J.L., 1942. The efficiency of three methods of selection. J. Hered., 33, 393399.

IHм P., 1959. Numerical evaluation of certain multivariate integrals. Sankhya, 21, 363-366.

JAIN J.P., Amble V.N., 1962. Improvement through selection at successive stages. J. Indian Soc. Agric. Stat., 14, 88-109.

Johnson N.L., Kotz S., 1972. Distributions in statistics : Continuous multivariate distributions. 333 pp., John Wiley \& Sons, New York.

Kendall M.G., 1941. Proof of relations connected with the tetrachoric series and its generalization. Biometrika, 32, 196-198.

Kendall M.G., Stuart A., 1945. The Advanced Theory of Statistics, vol. I. 433 pp., Hafner, New York.

Mardia K.V., Kent J.T., BibBy J.M., 1979. Multivariate Analysis. 521 pp., Academic Press, London.

MiLton R.C., 1972. Computer evaluation of the multivariate normal integral. Technometrics, 14, 881-889.

MiLton R.C., Hotchkiss R., 1969. Computer evaluation of the normal and inverse normal distribution functions. Technometrics, 11, 817-822.

Mineur H., 1966. Techniques de calcul numérique. 606 pp., Dunod, Paris.

Mukal F., Kitayama K., Fukushima T., 1985. Allocation of the selection intensity in two stage selection in order to achieve the genetic gains equivalent to selection index method. Jpn. J. Zootech. Sci., 56, 108-115.

Namkoong G., 1970. Optimum allocation of selection intensity in two stages of truncation selection. Biometrics, 26, 465-476.

Niebel E., Fewson D., 1976. Untersuchungen zur Zuchtplanung für die Reinzucht beim Zweinutzungsrind. II. Zuchtwahl in zwei Selektionsstufen. Z. Tierz. Züchtungsbiol., 93, 169-177.

OWen D.B., 1962. Handbook of statistical tables. 580 pp., Addison Wesley, Reading, Mass.

Patel J.K., Read C.B., 1982. Handbook of the normal distribution. 337 pp., Marcel Dekker, New York.

Plackett R.L., 1954. A reduction formula for normal multivariate integrals. Biometrika, 41, 351360.

Ralston A., Wilf H.S., 1967. Mathematical methods for digital computers, vol. II. 287 pp., John Wiley \& Sons, New York.

Robertson A., 1961. Inbreeding in artificial selection programmes. Genet. Res., 2, 189-194.

Saxton A.M., 1982. A note on a computer program for independent culling. Anim. Prod., 35, 295-297.

SIX F.B., 1981. Representations of multivariate normal distributions with special correlation structures. Commun. Stat., A10, 1285-1295.

Smith P., QuaAs R.L., 1982. Optimal truncation points for independent culling level selection involving two traits. Biometrics, 38, 975-980.

Tibau I Font J., Ollivier L., 1984. La sélection en station chez le porc. 69 pp., Bull. Tech. Dept. Genet. Anim. I.N.R.A., 37.

White J.S., 1970. Tables of normal percentile points. J. Am. Stat. Ass., 65, 635-638.

Young S.S.Y., 1961. A further examination of the relative efficiency of three methods of selection for genetic gain under less restricted conditions. Genet. Res., 2, 106-121. 


\section{Appendix 1}

\section{Detailed presentation of Dutt's method}

We want to compute Prob $\left(x_{1}>s_{1}, \ldots x_{n}>s_{n}\right)=L_{n}(s, R)$ :

$L_{n}(s, R)=\int_{s_{1}}^{+\infty} \ldots \int_{s_{n}}^{+\infty} f_{n}\left(x_{1}, \ldots x_{n}\right) d x_{1} \ldots d x_{n}=\int_{s}^{\infty} f_{n}(x) d x$

where $f_{n}(x)$ is the joint density of the $n$ normal variables. If these variables are standardized :

$$
\mathbf{f}_{\mathrm{n}}(\mathbf{x})=(2 \pi)^{-\mathrm{n} / 2}(\operatorname{det} \mathbf{R})^{-1 / 2} \exp \left(-1 / 2 \mathbf{x}^{\prime} \cdot \mathbf{R}^{-1} \cdot \mathbf{x}\right)
$$

where $\mathbf{R}$ is the correlation matrix.

1. Integration variable change using the characteristic function

By definition, the characteristic function $\varphi_{n}\left(t_{1}, \ldots t_{n}\right)$ with auxillary variables $t_{1}, \ldots t_{n}$ is equal to the expected value of $\exp \left(i\left(t_{1} x_{1}+\ldots+t_{n} x_{n}\right)\right)$

where $\mathrm{i}^{2}=-1$. In vector form :

$\varphi_{n}(t)=\int_{x=-\infty}^{x=+\infty} \exp \left(i \cdot t^{\prime} \cdot x\right) \cdot f_{n}(x) \cdot d x$

Conversely, a general theorem, the « inversion theorem » gives the expression of $f_{n}(x)$ as a function of $\varphi_{n}(t)$ (Kendall \& StUarT, 1945 ; Mardia et al., 1979) :

$f_{n}(x)=\frac{1}{(2 \pi)^{n}} \int_{t=-\infty}^{t=+\infty} \exp \left(-i \cdot t^{\prime} \cdot x\right) \cdot \varphi_{n}(t) \cdot d t$

In the particular case of the normal distribution, the characteristic function in equal to :

$$
\exp \left(\mathbf{i} \cdot \mathbf{t}^{\prime} \cdot \boldsymbol{\mu}-1 / 2 \cdot \mathbf{t}^{\prime} \cdot \mathbf{t} \cdot \mathbf{t}\right)
$$

where $\boldsymbol{\mu}$ is the vector of the expected values and $\$$ the variance-covariance matrix. With standardized variables, we have :

$$
\varphi_{\mathrm{n}}(t)=\exp \left(-1 / 2 \mathbf{t}^{\prime} \cdot \mathbf{R} \cdot \mathbf{t}\right)
$$

and therefore :

$f_{n}(x)=\frac{1}{(2 \pi)^{n}} \int_{t=-\infty}^{t=+\infty} \exp \left(-i \cdot t^{\prime} \cdot x-1 / 2 t^{\prime} \cdot R \cdot t\right) \cdot d t$

and $L_{n}(s, R)=\int_{x=s}^{\infty} \frac{1}{(2 \pi)^{n}} d x \int_{t=-\infty}^{t=+\infty} \exp \left(-i \cdot t^{\prime} \cdot x-1 / 2 t^{\prime} \cdot R \cdot t\right) \cdot d t$

$$
=\frac{1}{(2 \pi)^{n}} \int_{t=-\infty}^{t=+\infty} \exp \left(-1 / 2 t^{\prime} \cdot \mathbf{R} \cdot \mathbf{t}\right) \cdot \mathbf{d t} \int_{x=s}^{x=+\infty} \exp \left(-i \cdot t^{\prime} \cdot \mathbf{x}\right) \cdot d x
$$


after changing the order of integration. The last integral can also be written :

$\int_{x_{1}=s_{1}}^{\infty} \ldots \int_{x_{n}=s_{n}}^{\infty}\left[\prod_{j=1}^{n} \exp \left(-i \cdot t_{j} \cdot x_{j}\right)\right] \cdot d x_{1} \ldots d x_{n}$

$=\prod_{j=1}^{n} \int_{x_{j}=s_{j}}^{\infty} \exp \left(-i \cdot t_{j} \cdot x_{j}\right) \cdot d x_{j}$

$=\prod_{j=1}^{n}\left(-\frac{1}{i t_{j}}\right) \cdot\left[\exp \left(-i \cdot t_{j} \cdot x_{j}\right)\right]_{s_{j}}^{\infty}$

$=i^{-n} \cdot \prod_{j=1}^{n} \frac{\exp \left(-i \cdot t_{j} \cdot s_{j}\right)}{t_{j}}=\frac{i^{-n} \cdot \exp \left(-i \cdot t^{\prime} \cdot s\right)}{\prod_{j=1}^{n} t_{j}}$

and finally :

$\mathbf{L}_{\mathbf{n}}(\mathbf{s}, \mathbf{R})=(2 \pi \mathrm{i})^{-\mathbf{n}} \int_{t=-\infty}^{t=+\infty} \frac{\exp \left(-\mathrm{i} \cdot \mathbf{t}^{\prime} \cdot \mathbf{s}-1 / 2 \mathbf{t}^{\prime} \cdot \mathbf{R} \cdot \mathbf{t}\right)}{\prod_{j=1}^{n} t_{j}} d t$

This expression shows the interest of such a transformation. The integration variable $\mathbf{x}$ is substituted by a new variable $t$ for which the integration limits are no longer related to the initial truncation points, thus facilitating the use of known numerical methods. Moreover, if we apply a general decomposition theorem derived by GuRLAND (1948) to $L_{n}$, we obtain :

$L_{n}(s, \mathbf{R})=1 / 2+\left\{\sum_{m=1}^{n}(1 / 2)^{n-m} \underset{j_{1}<. .<j_{m}}{\sum} I_{m}\left(s_{j_{1}}, \ldots s_{j_{m}} ; r_{j_{1} j_{2}}, \ldots r_{j_{m-1} j_{m}} ; t_{j_{1}}, \ldots t_{j_{m}}\right)\right\}$

$\left(r_{j_{1} j_{2}}, \ldots r_{j_{m-1} j_{m}}\right.$ are the correlation coefficients between the variables $\left.j_{1}, \ldots j_{m}\right)$.

To simplify notation, let :

$\mathbf{s}_{\mathrm{m}}^{* \prime}=\left(s_{\mathrm{j}_{1}}, \ldots \mathrm{s}_{\mathrm{j}_{\mathrm{m}}}\right) ; \mathbf{t}_{\mathrm{m}}^{*}=\left(\mathrm{t}_{\mathrm{j}_{1}}, \ldots \mathrm{t}_{\mathrm{j}_{\mathrm{m}}}\right) ; \mathrm{t}_{\mathrm{m}_{\mathrm{j}}}^{*}$ is the $\mathrm{j}^{\text {th }}$ term of $\mathrm{t}_{\mathrm{m}}^{*}$

Then :

$I_{m}=$ Real part of $\left\{\lim _{\epsilon \rightarrow 0} \int_{\left|\mathbf{t}^{*}\right|>\epsilon} \frac{(2 \pi i)^{-m} \cdot \exp \left(-i \cdot t_{m}^{*} \cdot \mathbf{s}_{m}^{*}\right) \cdot \varphi_{m}\left(t_{m}^{*}\right) \cdot d t_{m}^{*}}{\prod_{j=1}^{m} t_{m j}^{*}}\right\}$

2. Reduction of the integration range to $(o,+\infty)$

This transformation is performed noting that, for any function $\mathrm{g}$ :

$\lim _{\epsilon \rightarrow 0} \int_{|t|>\epsilon}^{\infty} g(t) \cdot d t=\int_{0}^{\infty} \Delta(g(t)) \cdot d t \quad$ (DuTT \& Soms, 1976) 
where $\Delta(g(t))$ is the central difference of $g(t)$ about $t=0$. For example, for a simple integral :

$\int_{|t|>\epsilon}^{\infty} g(t) \cdot d t=\int_{\epsilon}^{\infty} g(t) \cdot d t+\int_{-\infty}^{\epsilon} g(t) \cdot d t=\int_{\epsilon}^{\infty} g(t) \cdot d t+\int_{\epsilon}^{\infty} g(-t) \cdot d(-t)=\int_{\epsilon}^{\infty} \Delta(g(t)) \cdot d t$

with $\Delta(g(t))=g(t)-g(-t)$, the central difference of order 1 .

By definition, the central difference of order $m$ is equal to :

$$
\begin{aligned}
\Delta_{m}\left[g\left(t_{1}, t_{2}, \ldots t_{m}\right)\right] & =g\left(t_{1}, t_{2}, \ldots t_{m}\right)-g\left(-t_{1}, t_{2}, \ldots t_{m}\right)-\ldots-g\left(t_{1}, t_{2}, \ldots-t_{m}\right) \\
& +(-1)^{2} \cdot g\left(-t_{1},-t_{2}, \ldots t_{m}\right)+\ldots+(-1)^{m} \cdot g\left(-t_{1},-t_{2}, \ldots-t_{m}\right)
\end{aligned}
$$

and then :

$$
I_{m}=\int_{0}^{\infty} \text { Real part of }\left\{\frac{(2 \pi i)^{-m} \cdot \Delta_{m}\left[\exp \left(-i \cdot t_{m}^{*} \cdot s_{n}^{*}\right) \cdot \varphi_{m}\left(t_{m}^{*}\right)\right]}{\prod_{j=1}^{m} t_{m_{j}}^{*}}\right\} \mathbf{d t}_{m}^{*}
$$

with :

$$
\begin{aligned}
\varphi_{\mathrm{m}}\left(\mathrm{t}^{*}\right) & =\exp \left(-1 / 2 \cdot \mathbf{t}_{\mathrm{m}}^{*^{\prime}} \cdot \mathbf{R}_{\mathrm{m}} \cdot \mathbf{t}_{\mathrm{a}}^{*}\right) \\
& =\exp \left(-1 / 2\left(\sum_{\mathrm{j}=1}^{\mathrm{m}} \mathrm{t}_{\mathrm{mj}}^{*}\right)\right) \cdot \exp \left(-\sum_{\mathrm{j} \neq \mathrm{j}^{\prime}} \mathbf{r}_{\mathrm{m} ; \mathrm{m}^{\prime}} \mathbf{t}_{\mathrm{m}_{\mathrm{j}}}^{*} \mathbf{t}_{\mathrm{m}_{\mathrm{j}}}^{*}\right)
\end{aligned}
$$

Furthermore, $\exp \left(-\mathrm{i} \cdot \mathbf{t}_{\mathrm{m}}^{*} \cdot \mathbf{s}_{\mathrm{m}}^{*}\right)$ is equal to :

$\cos \left(\sum_{j} t_{m_{j}}^{*} \cdot s_{m_{j}}^{*}\right)-i \cdot \sin \left(\sum_{j} t_{m_{j}}^{*} \cdot s_{m_{j}}^{*}\right)$

and then, it can be observed that :

$$
\Delta_{m}\left[-t_{m_{1}}, \ldots,-t_{m_{k}}, t_{m_{k}+1}, \ldots, t_{m_{n}}\right]=\Delta_{m}\left[t_{m_{1}}, \ldots, t_{m_{k}},-t_{m_{k+1}}, \ldots,-t_{m_{n}}\right]
$$

So, the evaluation of $I_{m}$ requires the computation of :

$\exp \left(-\mathrm{i} \cdot \mathbf{t}_{m}^{t_{m}^{\prime}} \cdot \mathbf{s}_{m}^{*}-1 / 2 \cdot \mathbf{t}_{m}^{*} \cdot \mathbf{R}_{m} \cdot \mathbf{t}_{m}^{*}\right)$ at $2^{m-1}$ points.

3. Numerical computation of the integrals $I_{\mathrm{m}}$ using Gauss-Hermite quadrature

According to the previous equalities, we have to compute :

$$
I_{m}=2(2 \pi)^{-m} \int_{0}^{\infty} \frac{\exp \left(-1 / 2 \cdot t_{m}^{*} \cdot t_{m}^{*}\right) \cdot D_{m}\left(t_{m}^{*}\right)}{\prod_{j=1}^{m} t_{m_{j}}^{*}} \cdot{d t_{m}^{*}}^{*}
$$

where $D_{m}$ is the sum of the first $2^{m-1}$ terms of the central difference of order $m$ of the real part of :

$$
\mathrm{i}^{-\mathrm{m}} \cdot \exp \left(-\mathrm{i} \cdot \mathbf{t}_{\mathrm{m}}^{*} \cdot \mathbf{s}_{\mathrm{m}}^{*}\right) \cdot \exp \left(-1 / 2 \cdot\left(\mathbf{t}_{\mathrm{m}}^{*} \cdot \mathbf{R}_{\mathrm{m}} \cdot \mathbf{t}_{\mathrm{m}}^{*}-\mathbf{t}_{\mathrm{m}}^{*} \cdot \mathbf{t}_{\mathrm{m}}^{*}\right)\right)
$$

In the expression of $D_{m}$, one can recognize the base function $\exp \left(-t^{2} / 2\right)$ for which a powerful integration method exists : the Gauss-Hermite quadrature. According to this method:

$\int_{-\infty}^{+\infty} \exp \left(-t^{2} / 2\right) \cdot g(t) \cdot d t \approx \sum_{k=1}^{n^{\prime}} w_{k} \cdot g\left(h_{k}\right)$ 
where the $h_{k}$ 's are the $n^{\prime}$ roots of the Hermite polynomial of degree $n^{\prime}$. The $w_{k}$ 's are integrals computed in such a way that strict equality holds when $g(t)$ is any polynomial of equal or lesser degree than 2n' 1 (AtKInSON, 1978 ; Booth, 1957 ; MineUR, 1966 ; BaKhvalov, 1976). In our case, an odd $n^{\prime}$ must be avoided : 0 is then one of the roots with $g(0)=+\infty$ and only the positive roots are considered since the integration range is $(0,+\infty)$. Then $h_{k}$ is the $k^{\text {th }}$ positive root of the Hermite polynomial of degree $2 n^{\prime}$ and $w_{k}$ is its associated weighting factor. The $h_{k}$ 's and $w_{k}$ 's (divided by $\sqrt{2}$ ) are tabulated in Abramowrtz \& Stegun (1972).

After $n$ successive quadratures, $I_{m}$ becomes :

$I_{m}=\frac{2}{(2 \pi)^{m}} \sum_{k_{1}=1}^{n^{\prime}} \ldots \sum_{k_{m}=1}^{n^{\prime}}\left(\frac{W_{k_{1}} \ldots W_{k_{m}}}{h_{k_{1}} \ldots h_{k_{m}}}\right) \cdot D_{m}\left(\left(h_{k_{1}} \ldots h_{k_{m}}\right)\right)$

$n^{\prime m} D_{m}$ functions have to be evaluated, each one being the sum of $2^{m-1}$ products of an exponential and a trigonometric function.

4. Formulae for the dimensions 1 to 3

Let $\lambda_{k}=w_{k} / h_{k}$

$L_{1}\left(s_{1}\right)=\frac{1}{2}+\frac{1}{\pi} \cdot \sum_{i=1}^{n^{\prime}} \lambda_{i} \cdot D_{1, i}\left(s_{1}\right)$

$L_{2}\left(s_{1}, s_{2} ; r_{12}\right)=\frac{1}{4}+\frac{1}{2 \pi} \sum_{u=1}^{2} \sum_{i=1}^{n^{\prime}} \lambda_{i} \cdot D_{1, i}\left(s_{u}\right)$

$$
+\frac{1}{2 \pi^{2}} \sum_{i=1}^{n^{\prime}} \sum_{j=1}^{n^{\prime}} \lambda_{i} \cdot \lambda_{j} \cdot D_{2 . i . j}\left(s_{1}, s_{2}\right)
$$

$\mathrm{L}_{3}\left(\mathrm{~s}_{1}, \mathrm{~s}_{2}, \mathrm{~s}_{3} ; \mathrm{r}_{12}, \mathrm{r}_{13}, \mathrm{r}_{23}\right)=\frac{1}{8}+\frac{1}{4 \pi} \sum_{\mathrm{u}=1}^{3} \sum_{\mathrm{i}=1}^{\mathrm{n}^{\prime}} \lambda_{\mathrm{i}} \cdot \mathrm{D}_{1, \mathrm{i}}\left(\mathrm{s}_{\mathrm{u}}\right)$

$$
\begin{aligned}
& +\frac{1}{4 \pi^{2}} \sum_{u=1}^{2} \sum_{v>u}^{3} \sum_{i=1}^{n^{\prime}} \sum_{j=1}^{n^{\prime}} \lambda_{i} \cdot \lambda_{j} \cdot D_{2, i, j}\left(s_{u}, s_{v}\right) \\
& +\frac{1}{4 \pi^{3}} \sum_{i=1}^{n^{\prime}} \sum_{j=1}^{n^{\prime}} \sum_{1=1}^{n^{\prime}} \lambda_{i} \cdot \lambda_{j} \cdot \lambda_{1} \cdot D_{3, i, j, l}\left(s_{1}, s_{2}, s_{3}\right)
\end{aligned}
$$

with :

$$
\begin{aligned}
& D_{1, j}\left(s_{u}\right)=-\sin \left(h_{i} \cdot s_{u}\right) \\
& D_{2, i, j}\left(s_{u}, s_{v}\right)=-\exp \left(-r_{u v} \cdot h_{i} \cdot h_{j}\right) \cdot \cos \left(h_{i} \cdot s_{u}+h_{j} \cdot s_{v}\right) \\
& \quad+\exp \left(r_{u v} \cdot h_{i} \cdot h_{j}\right) \cdot \cos \left(-h_{i} \cdot s_{u}+h_{j} \cdot s_{v}\right) \\
& \quad D_{3, i, j, t}\left(s_{1}, s_{2}, s_{3}\right)= \\
& \quad \exp \left(-r_{12} \cdot h_{i} \cdot h_{j}-r_{13} \cdot h_{i} \cdot h_{1}-r_{23} \cdot h_{j} \cdot h_{1}\right) \cdot\left[\sin \left(h_{i} \cdot a_{1}+h_{j} \cdot a_{2}+h_{1} \cdot a_{3}\right)\right] \\
& -\exp \left(r_{12} \cdot h_{i} \cdot h_{j}+r_{13} \cdot h_{i} \cdot h_{1}-r_{23} \cdot h_{j} \cdot h_{1}\right) \cdot\left[\sin \left(-h_{i} \cdot a_{1}+h_{j} \cdot a_{2}+h_{i} \cdot a_{3}\right)\right] \\
& -\exp \left(r_{12} \cdot h_{i} \cdot h_{j}-r_{13} \cdot h_{i} \cdot h_{1}+r_{23} \cdot h_{j} \cdot h_{1}\right) \cdot\left[\sin \left(h_{i} \cdot a_{1}-h_{j} \cdot a_{2}+h_{1} \cdot a_{3}\right)\right] \\
& -\exp \left(-r_{12} \cdot h_{i} \cdot h_{j}+r_{13} \cdot h_{i} \cdot h_{1}+r_{23} \cdot h_{j} \cdot h_{1}\right) \cdot\left[\sin \left(h_{i} \cdot a_{1}+h_{j} \cdot a_{2}-h_{i} \cdot a_{3}\right)\right]
\end{aligned}
$$




\section{Appendix 2}

\section{Detailed presentation of Deak's method}

Here we want to estimate Prob $\left(x_{1}<s_{1}, \ldots x_{n}<s_{n}\right)$ where the $x_{i}$ 's are standardized normal variables.

\section{a) General principle}

The vector $\mathbf{x}$ of correlated standardized normal variables can be expressed as a function of a vector $\mathbf{y}$ of independent standardized normal variables :

$$
\mathbf{x}=\mathbf{T} \cdot \mathbf{y}
$$

The matrix $\mathbf{T}$ is then a lower triangular matrix obtained by Cholesky decomposition of the correlation matrix $\mathbf{R}$ :

$$
\mathbf{T} \cdot \mathbf{T}^{\prime}=\mathbf{R}
$$

Under these conditions, $\sum_{j=1}^{n} y_{j}^{2}$ follows a $\chi_{n}^{2}$ distribution. With the additional transformation :

$\left.y_{j}^{*}=y_{j} / \sqrt{\left(\sum_{j=1}^{n} y_{j}^{2}\right.}\right) \quad\left(\sum_{j=1}^{n} y_{j}^{* 2}=1:\right.$ the extremity of $y^{*}$ moves over a hypersphere of radius 1$)$

it ensues that, if $y_{j}^{*}$ can be obtained in some way, $y_{j}$ is equal to $K \cdot y_{j}^{*}$ with $K$ following a $\sqrt{\chi_{n}^{2}}$ distribution. Then :

$$
\begin{aligned}
\mathrm{p} & =\operatorname{Prob}(\mathbf{x}<\mathbf{s}) \\
& =\operatorname{Prob}\left(\sqrt{\chi_{\mathrm{n}}^{2}} \mathbf{T} \cdot \mathbf{y}^{*}<\mathbf{s}\right) \\
& =\mathrm{E}\left(\sum_{\mathrm{y}^{*}} \frac{\operatorname{Prob}\left(\sqrt{\chi_{\mathrm{n}}^{2}} \mathbf{T} \cdot \mathbf{y}^{*}<\mathbf{s} \mid \mathbf{y}^{*}\right)}{\mathrm{N}_{\mathrm{y}}}\right)
\end{aligned}
$$

where $N_{y}$ is the total number of vectors $\mathbf{y}^{*}$ considered.

\section{Let $\mathbf{z}=\mathbf{T} \cdot \mathbf{y}^{*}$}

If $j_{+}$(respectively $j_{-}$) is the set of indices corresponding to positive (respectively negative) elements of $\mathbf{z}$, the conditional probability $p \mid \mathbf{y}^{*}$ is equal to the probability of realization of the following inequality :

$$
\begin{gathered}
0 \leqslant \max \left(s_{j_{-}} / z_{j_{-}}\right) \leqslant \sqrt{x_{n}^{2}} \leqslant \min \left(s_{j_{+}} / z_{j_{+}}\right) \\
\left(=m_{1}\right) \quad\left(=m_{2}\right)
\end{gathered}
$$

This probability is zero if $\mathrm{m}_{1}>\mathrm{m}_{2}$ or if $\mathrm{m}_{2}<0$. Otherwise, it is equal to $F_{n}\left(m_{2}^{2}\right)-F_{n}\left(m_{1}^{2}\right)$ where $F_{n}$ is the cumulative $\chi_{n}^{2}$ distribution. Therefore, a numerical algorithm with the greatest precision possible is required for the computation of these cumulative distributions.

b) Method of reduction of the sampling variance (for a given number of random vectors generated)

1) Associating the vector $-\mathbf{y}^{*}$ to each vector $\mathbf{y}^{*}$, we can write : 
$\operatorname{Prob}\left(\sqrt{\chi_{n}^{2}} \mathbf{T} \cdot\left(-\mathbf{y}^{*}\right)<\mathbf{s}\right)=\operatorname{Prob}\left(-\sqrt{\chi_{\mathbf{n}}^{2}} \mathbf{T} \cdot \mathbf{y}^{*}<\mathbf{s}\right)$

and no new vector generation is needed. Then, the sum of the 2 conditional probabilities $p\left|\mathbf{y}^{*}+p\right|\left(-\mathbf{y}^{*}\right)$, whose expected value is $2 p$, is equal to the probability of realization of the inequality :

$$
m_{1} \leqslant \pm \sqrt{\chi_{n}^{2}} \leqslant m_{2}
$$

i.e. :

$$
0 \quad \text { if } \mathrm{m}_{2}<\mathrm{m}_{1}
$$

$F_{n}\left(m_{2}^{2}\right)-F_{n}\left(m_{1}^{2}\right)$ if $0 \leqslant m_{1} \leqslant m_{2}$

$F_{n}\left(m_{2}^{2}\right)+F_{n}\left(m_{1}^{2}\right)$ if $m_{1} \leqslant 0 \leqslant m_{2}$

$-F_{n}\left(m_{2}^{2}\right)+F_{n}\left(m_{1}^{2}\right)$ if $m_{1} \leqslant m_{2} \leqslant 0$

2) The vectors $y$ are randomly generated in groups of $n$.

Each group is then transformed into an orthonormal base $B$ from which 4. $[n(n-1) / 2]=2 n(n-1)$ dependent normalized vectors can be drawn.

These vectors are of the following type :

$\sum_{j=1, j \neq j^{\prime}}^{n}\left[\left( \pm b_{j}\right)+\left( \pm b_{j^{\prime}}\right)\right] / \sqrt{2}$

According to what was presented in the previous section, only half of these vectors need to be considered, the second half being derived from the first one by sign change. These $n(n-1)$ vectors are then of the type :

$\sum_{j<j^{\prime}}\left(b_{j}+b_{j^{\prime}}\right) / \sqrt{2}$ or $\sum_{j<j^{\prime}}\left(b_{j}-b_{j^{\prime}}\right) / \sqrt{2}$

If $\mathbf{N}$ groups of vectors $\mathbf{y}$ are generated, we have :

$\hat{p}=\sum_{g=1}^{N}\left[\left(\sum_{j<j^{\prime}} p \mid \frac{b_{j}+b_{j^{\prime}}}{\sqrt{2}}\right)+\left(\sum_{j<j^{\prime}} p \mid \frac{b_{j}-b_{j^{\prime}}}{\sqrt{2}}\right)\right] / 2 N \cdot n(n-1)$

Using the notation used in a), we have therefore $N_{\gamma}=2 N \cdot n \cdot(n-1)$.

The sampling variance corresponding to this estimate was not theoretically evaluated by DEAK. However, empirical variances for the numerical applications studied were given (DEAK, 1980). 